\title{
HOW SHOULD WE IMPROVE THE RAY-TRACING METHOD?
}

\author{
B. V. BUDAEV
}

Dedicated to V. M. Babich

\begin{abstract}
The possibility is discussed to improve the ray approximation up to an exact representation of a wave field by the Feynman-Kac probabilistic formula (this formula gives an exact solution of the Helmholtz equation in the form of the expectation of a certain functional on the space of Brownian random walks). Some examples illustrate an application of the solutions obtained to diffraction problems.
\end{abstract}

It would not be an exaggeration to claim that a large (if not a major) part of studies in the theory of wave propagation is related somehow to the ray-tracing method. The contribution of Babich and Buldyrev to the creation of this method was quite substantial.

The ray-tracing method is based on the universal "divide and conquer" principle, which is revealed, in the present case, in splitting a difficult problem into two simpler ones. For instance, a solution of the Helmholtz equation

$$
\nabla^{2} \varphi+p^{2} k^{2}(x) \varphi=0, \quad p=\text { const },
$$

is sought in the form

$$
\varphi(x)=u(x) e^{i p s(x)}
$$

i.e., an unknown function $\varphi(x)$ is expressed in terms of two unknown functions $u(x)$ and $s(x)$. It is easily seen that the new unknowns must satisfy the equation

$$
\nabla^{2} u+2 i p \nabla s \nabla u+\left(i p \nabla^{2} s+p^{2} k^{2}-p^{2}(\nabla s)^{2}\right) u=0,
$$

which does not seem to be simpler than (1) but, in recompense, can be split in the eikonal equation

$$
(\nabla s)^{2}=k^{2}
$$

and the transport equation

$$
\frac{1}{2 p i} \nabla^{2} u+A \nabla u+B u=0
$$

with the coefficients

$$
A=\nabla s, \quad B=\frac{1}{2} \nabla^{2} s,
$$

determined by (3)

The procedure described above is feasible because the eikonal equation (3) can be solved exactly with the use of standard methods of Hamiltonian mechanics, which determine $s(x)$ uniquely on a certain (maybe, many-sheeted) domain $\widetilde{G}$ that covers the physical domain $G$. As to the transport equation (4), in distinction to the Helmholtz

2010 Mathematics Subject Classification. Primary 81Q30.

Key words and phrases. Diffraction, ray tracing method, stochastic equation, Feynman-Kac formula. 
equation (11) it admits a substantive approximation by a first order equation. Indeed, if $p \gg 1$, then equation (4) can be approximated by the equation

$$
A \nabla u_{0}+B u_{0}=0
$$

in the domain where $\left|\nabla^{2} u\right|<c$. Equation (5) can easily be solved in quadratures and leads to the first ray-tracing approximation

$$
\varphi \approx \varphi_{0}=u_{0} e^{i s} .
$$

Clearly, the same trick applied directly to the Helmholtz equation (11) yields the approximation $\varphi_{0}=0$, which is too crude to be informative.

The approximation (6) is the main result of the classical ray-tracing method; nearly all subsequent investigations dealt with attempts to improve the method, for example, by constructing the series

$$
u=u_{0}+\frac{1}{p} u_{1}+\frac{1}{p^{2}} u_{2}+\cdots
$$

whose terms are found one after another from equations of the form $A \nabla u_{n}+B u_{n}=$ $F\left(x, u_{0}, u_{1}, \ldots, u_{n-1}\right)$. So, the question arises as to whether it is possible to construct the exact solution of (5) by consecutive improvements of the first approximation (6).

At first glance, the answer is obviously in the positive, because the solution (which certainly exists) of the Helmholtz equation (11) can always be represented in the form (2) with a known eikonal $s(x)$, and the function $u(x)$ arising in this way may be viewed as an improvement of the first approximation $u_{0}(x)$ directly up to the exact solution (4). However, a closer look at the question makes the answer not so definitive.

Indeed, it is more natural to seek the solution of the Helmholtz equation (1) in the form

$$
\varphi=u_{+} e^{i p s}+u_{-} e^{-i p s},
$$

rather than in the form (2). In (7), the summands describe waves that propagate in different directions. Surely, (7) can always be reduced to the form (2) by putting

$$
u=u_{+}+u_{-} e^{-2 i p s},
$$

but in this case $u(x)$ acquires a rapidly oscillating component, which differs qualitatively from the first ray-tracing approximation and, consequently, cannot be represented by a rapidly convergent series (10).

Since a step-by-step improvement of the first ray-tracing approximation up to the exact solution seems inefficient, it is desirable to find an approach to the transport equation (4) which would yield the exact solution directly and, at the same time, would be as close as possible to the ray-tracing method in essence. It turns out that such an approach can be developed on the basis of the probabilistic approach to differential equations. We briefly discuss this paradigm.

It is well known (see [2]) that a fairly general boundary-value problem with nonconstant coefficients

$$
\sum_{n=1}^{N}\left(\frac{\sigma_{n}^{2}}{2} \frac{\partial^{2} u}{\partial x_{n}^{2}}+A_{n} \frac{\partial u}{\partial x_{n}}\right)+B u=0,\left.\quad u\right|_{\partial G}=f
$$

often admits an explicit solution given by the Feynman-Kac formula

$$
u(x)=E\left\{f\left(\xi_{t}\right) e^{\int_{0}^{\tau} B\left(\xi_{t}\right) d t}\right\} .
$$

Here $\xi_{t}=\left(\xi_{t}^{1}, \xi_{t}^{2}, \ldots, \xi_{t}^{N}\right)$ is the $N$-dimensional random walk described by the stochastic differential equations

$$
d \xi_{t}^{n}=\sigma\left(\xi_{t}\right) d \omega_{t}^{n}+A\left(\xi_{t}\right) d t, \quad \xi_{0}=x
$$


where $\omega_{t}=\left(\omega_{t}^{1}, \omega_{t}^{2}, \ldots, \omega_{t}^{N}\right)$ is the standard Brownian motion in the $N$-dimensional space, $\tau$ is the exit moment defined as the first value of $t$ at which $\xi_{t}$ hits the boundary $\partial G$, and $E$ is the operation of averaging (calculation of the mean value) over all possible Brownian trajectories $\omega_{t}$.

To obtain (10), it is convenient to apply Taylor's expansion

$$
u(x+\Delta x)=u(x)+\sum_{n=1}^{N}\left(\frac{\partial u}{\partial x_{n}} \Delta x_{n}+\frac{1}{2} \frac{\partial^{2} u}{\partial x_{n}^{2}}\left(\Delta x_{n}\right)^{2}\right)+\cdots
$$

to the case where the components of the vector increment $\Delta x$ are mutually independent random numbers

$$
\Delta x_{n}=A_{n} \varepsilon^{2} \pm \sigma_{n} \varepsilon, \quad 0<\varepsilon \ll 1 .
$$

Indeed, averaging of (11) over all possible values of $\Delta x_{1}$ (as in (12) ) brings equation (8) to the form

$$
E\{u(x+\Delta x)\}-\left(1-B \varepsilon^{2}\right) u(x)+o\left(\varepsilon^{2}\right)=0,
$$

whence we see that

$$
u(x)=E\left\{u(x+\Delta x) e^{B \varepsilon^{2}}\right\}+o\left(\varepsilon^{2}\right)
$$

the last formula represents $u(x)$ as a weighted average of the same function over neighboring points. Finally, applying (13) by recursion and putting $\Delta t=\varepsilon^{2}$, we arrive at (13).

First it should be noted that if (8) does not involve second derivatives, then the corresponding stochastic equations (10) reduce to ordinary differential equations

$$
d \xi_{t}^{n}=A_{n}\left(\xi_{t}\right) d t
$$

which determine motion along the vector field $A=\left(A_{1}, A_{2}, \ldots, A_{N}\right)$. As a result, the Feynman-Kac formula (9) reduces to the explicit solution

$$
u(x)=f\left(\xi_{\tau}\right) e^{\int_{0}^{\tau} B\left(\xi_{t}\right) d t}
$$

of the first order equation $A \nabla u+B u=0$, which is the basis of the ray method. This observation shows that probabilistic methods, when applied to the transport equation (5), do not contradict classical ray asymptotics, but upgrade them to the exact solution of the second order equation (4).

Another important feature of the Feynman-Kac formula (9) is the presence of the exponential $e^{\int_{0}^{\tau} B\left(\xi_{t}\right) d t}$, which "does not hamper anything for sure" only if $\operatorname{Re} B(x)<0$. If this inequality is violated, the expression in (9) may diverge or may define a rapidly oscillating function. For instance, a direct application of (9) to the Helmholtz equation $\nabla^{2} \varphi+k^{2} \varphi=0$ in a domain $G$ with Dirichlet boundary conditions yields a convergent expression if and only if $G$ is so small that its first eigenvalue does not exceed $k$. As another example, observe that if $B$ is purely imaginary and has sufficiently large modulus, then the function $u(x)$ defined by (9) may be rapidly oscillating.

Finally, in order that the Feynman-Kac formula (9) make sense, it is necessary that, eventually, the trajectories of the random walk $\xi_{t}$ hit the boundary $\partial G$ (where the values $u(x)$ are known from the boundary conditions). It is easily seen that this condition is a serious obstruction for application of (9) to resolving the transport equation (4), which involves the complex coefficient $1 / 2 p i$. Indeed, since the one-dimensional trajectory $\xi_{t}$ defined by equation (10) with complex coefficients may escape into the complex space of real dimension $2 N$, it may never intersect the $(2 N-2)$-dimensional analytic extension of the boundary $\partial G$. 
To overcome this difficulty, it often suffices to multiply the initial equation (44) by a specially chosen function $\gamma^{2}(x)$. Indeed, this leads to the equation

$$
\sum_{n=1}^{N}\left(\frac{\gamma^{2} \sigma_{n}}{2} \frac{\partial^{2} u}{\partial x_{n}^{2}}+\gamma^{2} A_{n} \frac{\partial u}{\partial x_{n}}\right)+B \gamma^{2} u=0,
$$

which has the same structure as (4) and, accordingly, can be solved by the formula

$$
u(x)=E\left\{f\left(\xi_{t}\right) e^{\int_{0}^{\tau} B\left(\xi_{t}\right) \gamma^{2}\left(\xi_{t}\right) d t}\right\},
$$

where the trajectories $\xi_{t}$ are determined by the stochastic differential equations

$$
d \xi_{n}=\sigma_{n}\left(\xi_{t}\right) \gamma\left(\xi_{t}\right) d \omega_{t}^{n}+A_{n}\left(\xi_{t}\right) \gamma^{2}\left(\xi_{t}\right) d t .
$$

If $\gamma\left(\xi_{t}\right)$ is real, equations (10) and (14) determine the same collections of trajectories, differing by a time scale only.

But if $\gamma\left(\xi_{t}\right)$ takes complex values, the structure of trajectories changes substantially. In particular, it turns out to be possible to choose $\gamma(x)$ so that the corresponding trajectories $\xi_{t}$ will be localized in some $(2 N-1)$-dimensional domain.

The above arguments show that probabilistic methods are applicable to the transport equation (4) in principle, which encourages us to search for ways of applying such a method to specific problems.

To illustrate the possibilities of the probabilistic approach to problems of diffraction, it suffices to analyze the two-dimensional Helmhholtz equation $\nabla^{2} \varphi+\varphi=0$ in the angular domain $|\theta|<\alpha$ with the boundary conditions $\varphi(r, \pm \alpha)=f_{ \pm \alpha}(r) e^{i r}$, where $f_{ \pm \alpha}(r)$ are analytic functions of the complex radius $r$.

Applying the method outlined above, we seek the solution $\varphi(r, \theta)$ in the form

$$
\varphi(r, \theta)=u(r, \theta) e^{i r}
$$

in which $u(r, \theta)$ satisfies the equation

$$
\frac{\partial^{2} u}{\partial r^{2}}+\frac{1}{r} \frac{\partial u}{\partial r}+\frac{1}{r^{2}} \frac{\partial^{2} u}{\partial \theta^{2}}+2 i \frac{\partial u}{\partial r}+\frac{i}{r} u=0
$$

and the boundary conditions $u(r, \pm \alpha)=f_{ \pm \alpha}(r)$.

It is easily seen that a direct application of (9) and (10) to equation (15) fails because the corresponding random walks have no chance to intersect the boundary $\theta= \pm \alpha$ of the angular domain. However, multiplication of (15) by $r^{2}$ leads to the equation

$$
\frac{r^{2}}{2} \frac{\partial^{2} u}{\partial r^{2}}+i r\left(r-\frac{i}{2}\right) \frac{\partial u}{\partial r}+\frac{1}{2} \frac{\partial^{2} u}{\partial \theta^{2}}+\frac{i u}{2}=0,
$$

which can be resolved by the formula

$$
u(r, \theta)=E\left\{f_{ \pm \eta_{\tau}}\left(\xi_{\tau}\right) e^{\frac{i}{2} \int_{0}^{\tau} \xi_{t} d t}\right\} ;
$$

here $\eta_{t}$ is the Brownian motion launched from the point $h_{0}=\theta$ and hitting the boundary of the interval $|\eta|<\alpha$ at the time moment $t=\tau$. As to the random walk $\xi_{t}$, it is described by the stochastic equation

$$
d \xi_{t}=\xi_{t} d \omega_{t}+\xi_{t}\left(\xi_{t}-i / 2\right) d t, \quad \xi_{0}=r,
$$

where $\omega_{t}$ is a standard Brownian motion independent of $\eta_{t}$. It is easily seen that the motion $\xi_{t}$ looks like a random walk inside the first quarter of the complex plane, with drift towards $\xi=i / 2$ (see Figure 1). Clearly, this localization of $\xi_{t}$ guarantees fast convergence in (16).

Surely, to apply formula (16), it is necessary that the boundary values of $f_{ \pm \alpha}(r)$ be analytic in $r$. However, in diffraction problems this condition is always fulfilled, and the procedure described above makes it possible to solve many nontrivial problems. For 


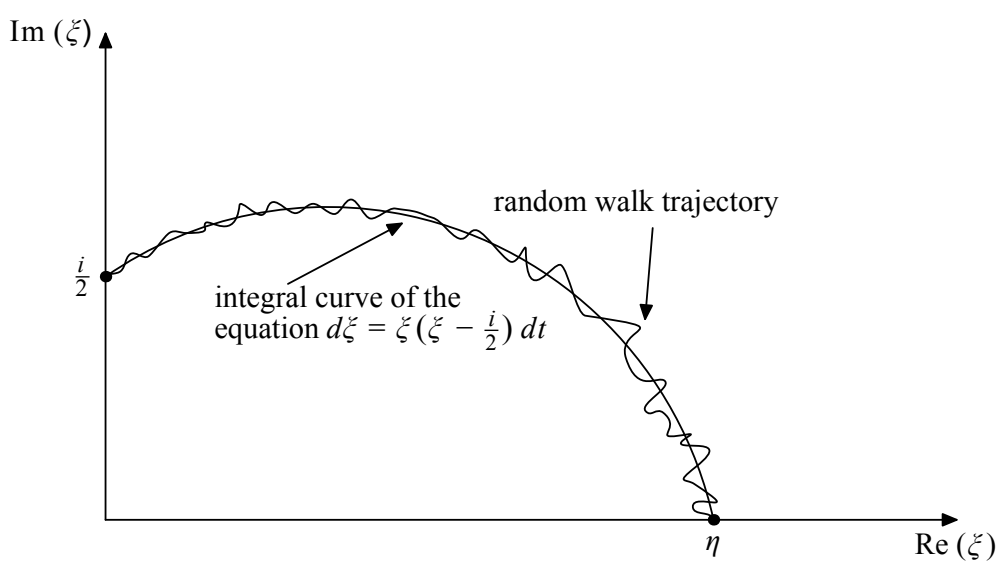

FIgURE 1. Example of a random walk determined by equation (17).

instance, in [3, there are references to a solution of the Maluizhinets problem of diffraction by a wedge, the diffraction problem on an arbitrary polygon, the three-dimensional problem of diffraction on a plane sectorial screen, as well as a more general problem of diffraction on a pyramid of infinite length. Moreover, it is possible to solve the problems mentioned above not only for Dirichlet and Neumann boundary conditions, but also for much more general conditions of impedance type.

It should be noted that the probabilistic approach enables us not only to solve diffraction problems on bodies of simple geometry, but also problems of wave propagation in nonhomogeneous media. In the latter, an important part is played by waves reflected backwards, whose calculation is the Achilles heel of the classical ray-tracing method. For instance, exact solutions of the equation $\varphi^{\prime \prime}+x \varphi=0$ (which determines Airy functions) can be obtained in this way. Also, this approach is applicable to tunneling and above-barrier reflection problems, which are so important that they deserve a separate paper.

In conclusion, I would like to express my sincere gratitude to V. B. Babich; along with G. I. Petrashen', he played a crucial role in the formation of my scientific field of interest.

\section{REFERENCES}

[1] V. M. Babich and V. S. Buldyrev, Asymptotic methods in diffraction problems of short-length waves. The method of canonical problems, Nauka, Moscow, 1972; English transl., Short-wavelength diffraction theory. Asymptotic methods, Springer Series on Wave Phenomena, vol. 4, Springer-Verlag, Berlin, 1991. MR0426630 (54:14569), MR1245488(94f:78004)

[2] M. Freidlin, Functional integration and partial differential equations, Ann. of Math. Stud., No. 109, Princeton Univ. Press, Princeton, NJ, 1985. MR0833742 (87g:60066)

[3] B. Budaev and D. Bogy, Diffraction by a convex polygon with side-wise constant impedance, Wave Motion 43 (2006), 631-645. MR2267276 (2007h:35035)

Department of Mechanical Engineering, University of California at Berkeley, Berkeley, CALIFORNia 94720-1740

E-mail address: budaev@berkeley.edu

Received 7/SEP/2010

Translated by S. KISLYAKOV 